\title{
Established and experimental techniques to improve phalloplasty outcomes/optimization of a hypercomplex surgery
}

\author{
Erin E. Carter ${ }^{1}$, Curtis N. Crane ${ }^{2}$, Richard A. Santucci ${ }^{2}$ \\ 'Department of Urology, Boston University School of Medicine, Boston, MA 02119, USA. \\ ${ }^{2}$ The Crane Center for Transgender Surgery, Austin, TX 78746, USA.
}

Correspondence to: Dr. Richard A. Santucci, Senior Surgeon, The Crane Center for Transgender Surgery, 5656 Bee Cave Rd Suite J201, Austin, TX 78746, USA. E-mail: richard@cranects.com

How to cite this article: Carter EE, Crane CN, Santucci RA. Established and experimental techniques to improve phalloplasty outcomes/optimization of a hypercomplex surgery. Plast Aesthet Res2020;7:33. http://dx.doi.org/10.20517/2347-9264.2020.81

Received: 15 Apr 2020 First Decision: 18 May 2020 Revised: 27 May 2020 Accepted: 19 Jun 2020 Published: 30 Jun 2020

Science Editors: Marlon E. Buncamper, Stan J. Monstrey Copy Editor: Cai-Hong Wang Production Editor: Tian Zhang

\begin{abstract}
An increasing number of transgender and gender non-conforming patients are seeking genital gender affirming surgeries in order to better align their physical characteristics with their innate gender identity and treat gender dysphoria. Phalloplasty is the most complex of these surgeries, and this complexity creates a wide range of potential complications. Some of the most common complications and therefore, targets for improvement in outcomes, concern neourethral fistula/stricture, efficacy of reinnervation of the phalloplasty flap, postoperative flap monitoring, and donor site morbidity. In the setting of no established "gold standard", this review seeks to describe the components and staging of phalloplasty, with an emphasis on established and experimental solutions to the most common and vexing problems.
\end{abstract}

Keywords: Phalloplasty, transgender, female to male, transmasculine, surgical complications, radial forearm flap, anterolateral thigh flap, genital gender confirmation

\section{INTRODUCTION}

Gender dysphoria is the distress resulting from a marked incongruence between a patient's natal sex and their innate gender identity. Treatment of gender dysphoria in transgender (TG), non-binary, and gender non-conforming patients (TGNC) may include both medical and surgical interventions using shared decision making to customize treatment plans according to individual patient goals. A significant and growing population are seeking out genital gender-affirming surgery (gGAS) and an increasing number of surgical centers are available to meet that need ${ }^{[1,2]}$. For such patients, these procedures improve quality of life,

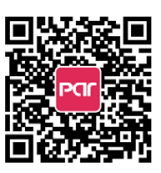




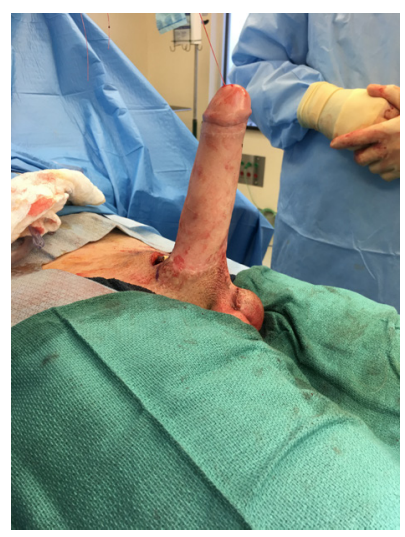

Figure 1. Excellent aesthetic results following phalloplasty and placement of erectile and testicular prostheses

relieve gender dysphoria and decrease suicidality ${ }^{[1-5]}$. Masculinizing gGAS usually involves some combination of removing natal female genital structures and creating functional natal male-type genitourinary structures. Phalloplasty (with or without simultaneous vaginectomy, urethral lengthening and scrotoplasty) is one such procedure that seeks to create an aesthetic and functional neophallus.

Phalloplasty uses existing analogous structures that share a common embryologic origin, as well as carefully harvested fasciocutaneous allograft(s), to create a neophallus. The goals are to create an aesthetic neophallus that has both tactile and erogenous sensation that can be directed for standing micturition, and has sufficient girth to accommodate penile prosthesis ${ }^{[6-9]}$. Erection and penetrative intercourse may be achieved, usually following penile prosthetic placement ${ }^{[6,7]}$.

Phalloplasty is an exceptionally complex reconstructive surgery. For instance, a single-stage phalloplasty (including vaginectomy, urethral lengthening and scrotoplasty) represents 200 relative value units (RVUs) of surgery (RVUs are a measure of value used in the United States which rank on a common scale the resources used to provide physician services). As a comparison, a craniotomy for removal of a glioblastoma multiforme brain tumor is 85 RVUs. Though this ultracomplex surgery may be daunting, excellent aesthetic and functional results with high patient satisfaction are achievable [Figure 1].

Surgical techniques used by gGAS surgeons for each component varies widely and are largely nonstandardized ${ }^{[4]}$. This article seeks to describe the components of phalloplasty as well as common complications and potential technical improvements that may be used to lower complications. Some targets for improvement in outcomes concern neourethral fistula/stricture, efficacy of reinnervation of the phalloplasty flap, postoperative flap monitoring, and donor site morbidity. Although several improvements in technique have occurred in recent years, ongoing innovation in surgical technique is necessary to further improve patient outcomes.

\section{THE QUESTION OF STAGING}

The components of phalloplasty include the creation of a penile shaft, penile urethroplasty, urethral lengthening (perineal urethroplasty), scrotoplasty, glansplasty, vaginectomy, hysterectomy, salpingoophorectomy (if desired), testicular implants, and erectile device implant ${ }^{[6,9]}$. The inclusion, staging, and order of these procedures may be altered to align with each patient's treatment goals as well as the surgeon's assessment of best practices. Each individual component, especially penile shaft creation and penile urethroplasty, have multiple surgical approaches.

The ideal phalloplasty would be a single-stage and reproducible surgery with minimal complications. However, because of the range, severity, and prevalence of complications of the surgery, some have elected to 
divide the procedure into multiple stages ${ }^{[10]}$. There is no consensus or "gold standard" among gGAS surgeons regarding the optimal number of stages or sequencing of reconstructive steps. Of note, our high-volume center generally does not stage phalloplasties except in unusual situations.

Centers like ours that usually perform phalloplasty, vaginectomy, scrotoplasty and urethral lengthening in a single stage have shown decreased rates of flap-related complications and increased patient satisfaction ${ }^{[10]}$. A major advantage to the single-stage approach is that most patients (up to $60 \%$ at our center) will require no further surgery until the time of penile prosthesis placement. We use a team approach to efficiently use operating room time such that a single-stage phalloplasty routinely takes about $6 \mathrm{~h}$, and seldom as much as $8 \mathrm{~h}$. Other centers favor a staged approach, citing the potential for fewer neourethral complications and more straightforward management of complications, even at the cost of more operations for these patients ${ }^{[4,9,10]}$. However, not all researchers have found that staging phalloplasties will result in fewer complications ${ }^{[4]}$. Even if staging ultimately proves to not be effective at decreasing complications, multiple staged procedures allow for sophisticated surgical planning. For example, hysterectomy may be consolidated into one of the phalloplasty stages. Regardless of staging choice, it is agreed that any prosthetic placement, including penile prosthesis and testicular prostheses should be performed in a later stage after complete healing has occurred and some sensation restored ${ }^{[6,8,11]}$.

\section{FLAP SELECTION, PREPARATION AND DONOR SITE HEALING}

When selecting a donor site for creation of the penile shaft and penile neourethra, it is important to consider the benefits and drawbacks of each potential site, as well as patient goals. All flaps must have sufficient innervation and vascularity to allow for microsurgical creation of the neophallus. The radial forearm flaps (RFF) are the most commonly used and most widely studied option ${ }^{[6,12]}$, followed by pedicled anterolateral thigh flaps (ALT $)^{[3,11]}$. RFF provides a robust, well vascularized and appropriately-sized neophallus in most cases, at the cost of requiring a microvascular anastomosis that may clot suddenly ( $<1 \%$ of cases). The RFF tends to be thinner (particularly important in patients with increased adiposity) and more pliable, and may be more similar to natal genital tissue ${ }^{[3,11]}$. The major downside of the RFF is removal of skin from an exposed and functionally important location on the body ${ }^{[13]}$. Some patients may have concerns about the visibility and potential recognizability of the resultant scar, and thus may want to avoid using a RFF flap ${ }^{[1,5,6]}$.

The ALT has the noted benefit of not usually requiring a microvascular anastomosis, and may have less risk of significant vascular occlusion emergencies as a result. However, the microcirculation of ALT flaps is less robust than RFF flaps, and a moderately higher chance of partial flap loss is well described with ALT flap use $^{[9,12]}$. It is said that in some cases, the inherent rigidity of the ALT flap may allow for sufficient rigidity for penetrative intercourse without the need for an additional prosthetic device, although most of our patients still require the addition of a penile prosthesis for penetrative intercours $\mathrm{e}^{[3,6]}$. The resultant scarring at the donor site may be less distressing and easier to hide for ALT flaps.

Less common flap donor sites include abdominal flaps, modified latissimus dorsi flaps (MLD), tibial free flaps, and superficial circumflex artery perforator flaps ${ }^{[3]}$. Of these, only the MLD is frequently chosen today.

\section{Preoperative hair removal}

The consensus is that donor flaps should be hairless, either innately or following removal methods such as laser hair removal or electrolysis. However, the efficacy and long-term permanence of these methods is poorly studied. While preoperative hair removal likely decreases the number and thickness of hairs, it is unlikely that the results of even the most thorough hair removal protocol are ever truly permanent ${ }^{[3,14]}$. Many phalloplasty patients have some hair regrowth in the neourethra ${ }^{[9,14,15]}$. Most patients are unaffected by urethral hair, but complications including infection, calculi, and trichobezoars/hairballs can occur, although uncommon ${ }^{[15,16]}$. Urinary obstruction from neourethral hair [Figure 2] can happen despite thorough 


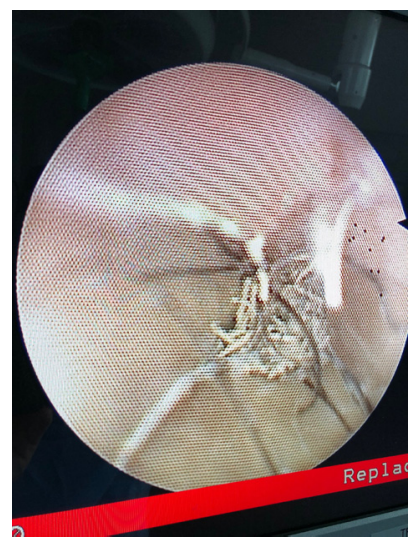

Figure 2. Cystoscopic appearance of abundant hair in the proximal penile urethra, which became calcified by urinary solutes and created an obstructive intraurethral trichobezoar/hairball

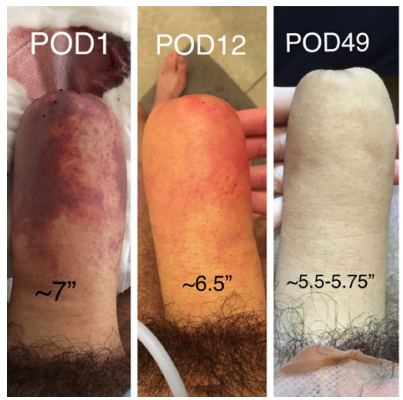

Figure 3. Comparison of penile girth after liposuction by postoperative day (POD), showing a $20 \%$ decrease in girth over time. Of note, there is prominent ecchymosis and swelling immediately after the procedure, which subsides with time

preoperative depilitation ${ }^{[14]}$. While we continue to recommend thorough preoperative depilitation, especially of the neourethral portion of the future flap, the optimal hair removal protocol has yet to be devised.

\section{Implications of donor site adiposity}

Donor site individualization based on patient preference is our practice. However, certain body habitus types may have more favorable outcomes by choosing one flap type over another. For instance, particularly thin individuals should avoid the RFF because the resultant neophallus may be disproportionately thin. Conversely, these thin individuals tend to do especially well with ALT flaps, as the donor site will not be too thick for creating an aesthetic and proportionate neophallus. Individuals with increased adiposity of the anterior thigh should avoid ALT flaps if possible and may do particularly well with a RFF flap instead ${ }^{[3]}$. We advise our patients of these considerations before they finalize their choice of donor site.

Especially thick ALT flaps create several problems. The first is that the resultant neophallus may be disproportionately large in circumference. Contouring these very large neophalluses down to an aesthetic, biologically-appropriate size may be difficult or even impossible. Most ALT flap patients will require one liposuction session to reduce penile girth [Figure 3]. However, some particularly large ALT neophalluses may require multiple liposuction procedures, open surgical reduction of ventral penile skin to decrease girth of the shaft, and contouring of the base of the neophallus to create a more proportional outcome. Even with multiple revision surgeries, excellent aesthetic results may never be achievable.

The second major problem with too-thick ALT flaps is the possibility that the neourethra cannot be made in the first stage. Using the tube-within-a-tube (TWT) technique, it may be impossible to close a particularly 


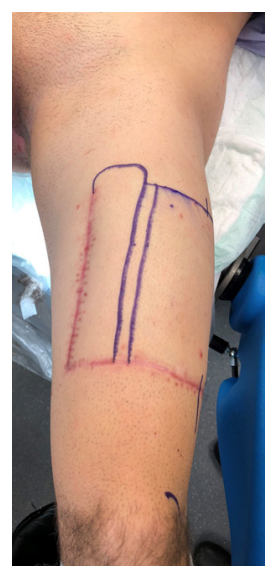

Figure 4. Delayed anterolateral thigh flap ready for use for phalloplasty, 6 months after flap creation. Note that the superior contour of the flap has not been incised in order to decrease flap edema. Nonviable flap edges (not present in this patient) can be identified and removed if present at this stage

thick flap to create the neophallus if the flap thickness is too great ${ }^{[3]}$. In that case, we do not tubularize the flap to create the neourethra in the first stage and instead, perform penile urethroplasty in a subsequent stage. Staged penile urethroplasty is a long, invasive procedure that may have a higher rate of dehiscence than primary urethroplasty ${ }^{[6,10]}$. We counsel and caution our patients when pinched anterior thigh skin/fat thickness is $>1 / 2$ inch $(1.3 \mathrm{~cm})$ and strongly advise against the ALT flap in those with a pinched thigh skin/fat thickness $>1$ inch $(2.5 \mathrm{~cm})$.

The ideal management of the too-thick ALT donor site, other than avoidance of this flap, has not been established. We have created an experimental protocol for patients with thick ALT flap donor sites who request ALT phalloplasty and will not/cannot consider RFF phalloplasty. The surgery is staged, allowing for two opportunities to thin the flap while protecting the blood supply in the tissue to be used to make the neourethra and shaft. We perform a simultaneous subtotal vaginectomy and delay of the flap in the first stage. The flap is dissected in the usual fashion, except the tissue around its perforators is left unoperated. Fat deep to Scarpa's fascia is removed, which moderately reduces phallic girth. The superior incision line is not created at this time in order to decrease subsequent lymphedema of the flap [Figure 4]. After 6 months, there is compensatory hypertrophy of the perforators, any tissue loss at the edge of the flap will have occurred and is discarded, and a second moderate thinning of the flap possible. We do not thin the flap during the typical single-stage ALT phalloplasty because of the increased risk of devascularizing the flap.

\section{Optimizing the donor template}

Donor site dimensions are important when considering the final length and girth of the neophallus, including sufficient girth for the insertion of a penile prosthetic device. Depending on the anticipated surgical technique, the flap design may include the penile shaft only, two separate flaps for the penile shaft and penile urethra, or the TWT flap design. The TWT flap style is a well-established technique and is almost exclusively used in our high-volume center when urethral extension is desired. The ideal donor site configuration is unknown, but we have introduced several small modifications that appear to improve outcomes.

(1) Create an "as short-as-possible" urethral extension. This allows the neourethra to protrude from the shaft, simplifying urethral anastomosis. This urethral extension can theoretically impair blood flow to this portion of the flap, so we create the shortest possible extension that will allow for tensionless anastomosis.

(2) In order to maximize the proximal urethral blood supply, we spare the dermis medial to the urethral extension as well as a $2-3 \mathrm{~cm}$ zone of subdermal fat superior to the urethral extension. 


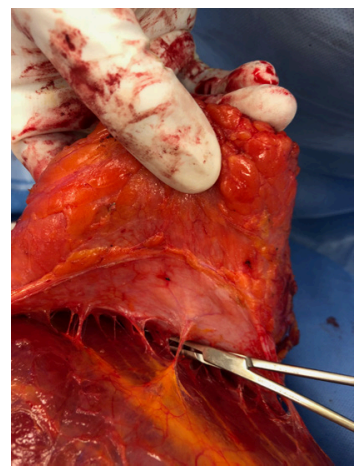

Figure 5. Intraoperative display of small anterolateral thigh flap perforators, measuring $<1 \mathrm{~mm}$ in diameter

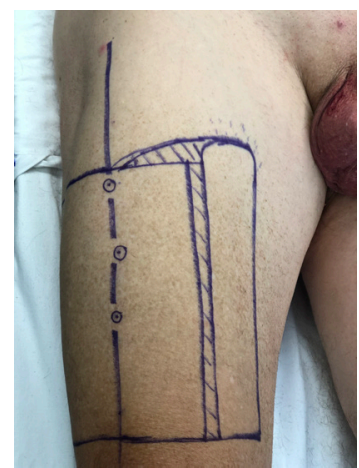

Figure 6. Preoperative marking of an anterolateral thigh flap donor site showing (1) as short-as-possible urethral extension; (2) deepithelialization of the proximolateral dermis next to the urethral extension; (3) sparing of the fat around the urethral extension. Circled dots represent the location of three flap perforators identified with a handheld Doppler

(3) The vascularity of an ALT flap relies on the patient's anatomy, specifically the perforators arising from the descending branch of the lateral circumflex femoral artery ${ }^{[3,9]}$. It is advantageous to include the largest and as many perforators as possible when designing this particularly large flap ${ }^{[9]}$. When using the ALT flap, we use a handheld Doppler to mark the patient's dominant perforators while the patient is awake in the preoperative holding area. An example of particularly small ALT perforators is shown in Figure 5.

(4) When using the ALT flap, we advise the patient to stop injecting testosterone into the donor thigh. Common formulations of depo testosterone contain benzyl benzoate, benzyl alcohol, and a large amount of cottonseed oil (736/100 mg of testosterone), creating a nidus of scar, which is best avoided in flap donor sites. In fact, we advocate that standard instructions for intramuscular testosterone injections should be modified from "switch thigh injection sites from side to side with each injection" to "pick a side and stick to it" in a patient who thinks they may ever be interested in ALT phalloplasty.

Figure 6 illustrates the preoperative marking of an ALT donor site from our center highlighting these configuration modifications.

We are interested in using imaging technology, perhaps CT angiogram, as some other groups have reported, to select the thigh with the most robust perforator blood supply in the future ${ }^{[\sigma, 9]}$. However, this has not currently been shown to be effective in improving outcomes ${ }^{[17]}$.

\section{RFF donor site coverage}

Once a fasciocutaneous RFF flap is removed, the underlying tendons, muscles, and nerve are exposed. Historically, the RFF donor site has been managed with a single layer split thickness skin graft (STSG ${ }^{[18]}$. 


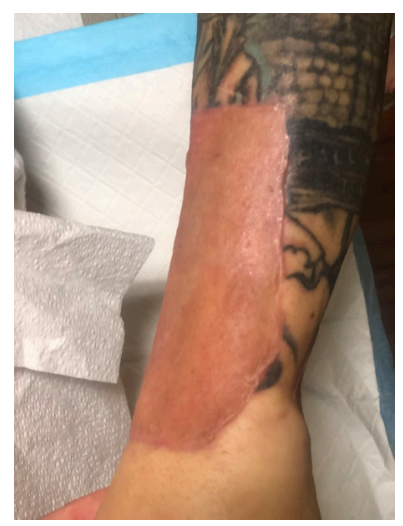

Figure 7. Six-week postoperative appearance of radial forearm flap donor site covered with thin Integra ${ }^{\circledR}, 18 / 1000$-inch thick split thickness skin graft, and negative pressure wound therapy for 8 days. Note excellent take of the skin graft, minimal step-off, and plump, nonadherent configuration of the graft

However, using an STSG alone can be associated with significant donor site morbidity ${ }^{[11,18]}$. STSGs may adhere to the exposed tendons and reduce the strength and range of motion, lead to contractures, and is frequently distressing to the patient ${ }^{[13,18]}$.

Recently, we and others have been using artificial dermal substitutes such as Integra ${ }^{\bullet}$ Wound Matrix (Thin) (Integra LifeSciences; Plainsboro, NJ) to improve RFF donor site healing and cosmesis ${ }^{[6,13,18]}$. Integra $^{\circ}$ is a synthetic acellular dermal regeneration template that is composed of a bilaminate sheet of cross-linked bovine tendon collagen coated with shark glycosaminoglycans (chondroitin-6-sulfate) ${ }^{[19]}$. Originally developed for the coverage of burn wounds, Integra ${ }^{\circledR}$ provides a scaffolding for revascularization and growth of the neodermis while creating a gliding surface against the underlying nerve, muscle, and tendon ${ }^{[19]}$. We place the thin Integra ${ }^{\circ}$ over the wound, followed by an 18/1000-inch unmeshed STSG, and then apply negative pressure wound therapy with a V.A.C ${ }^{\mathrm{TM}}$ device (KCI; San Antonio, TX) for at least 9 days postoperatively, and sometimes up to 12 days. We have found that this method of single-stage Integra ${ }^{\circ}$ and immediate STSG application has equivalent graft take results as the staged approach. Though a staged approach is the most common, single-stage Integra ${ }^{\circledR}$ application has been described by others in a variety of applications previously ${ }^{[20-23]}$.

We have found that using Integra ${ }^{\bullet}$ under the usual STSG in this way results in improved cosmesis with a noticeably thicker result and less step-off. It also minimizes the risk of tight adhesion of the STSG to the underlying deep arm structures ${ }^{[13,18]}$. Other groups have also documented improvement in skin elasticity, motor function, range of movement, wound contractures and hypertrophic scar formation with Integra ${ }^{[13,18]}$. Figure 7 displays one of our patient's RFF donor site managed with thin Integra ${ }^{\oplus}$, STSG, and negative pressure wound therapy at six weeks after surgery.

\section{URETHRAL LENGTHENING COMPLICATIONS}

The most common complications of phalloplasty involve the urethral lengthening portion of the procedure. In recent years, improved surgical technique has greatly decreased the rate of neourethral complications, but all-cause urinary complications from phalloplasty are still reported to be between $35 \%-58 \%{ }^{[4,8]}$. The rate of complications is so high that patients should decide if urinating from the tip of the neophallus is imperative for their treatment goals, as maintaining the external urethral orifice in the native position and forgoing standing voiding forever greatly reduces the risk of urinary complications ${ }^{[6]}$.

In general, urinary strictures tend to have significant morbidity, and open surgical intervention is required in most (94\%-96\%) stricture patients ${ }^{[24]}$. The management options of neourethral stricture after phalloplasty 
are the same as those for native male urethral repair ${ }^{[11,24]}$. Unfortunately, these repairs have a high predicted failure rate, reported as high as $50 \%$ in this population ${ }^{[24]}$. In some cases, especially those requiring multiple surgical interventions, a temporary or permanent perineal urethrostomy may be necessary ${ }^{[24]}$. Patients generally are at risk for stricture formation for at least 1 year, although most strictures present within the first 6 months after phalloplasty ${ }^{[11]}$.

Fistulas have overall lower morbidity than strictures. 17\%-35\% of fistulas may heal spontaneously within 3 months without the need for surgical intervention ${ }^{[24]}$. If spontaneous recovery does not occur, surgical repair has a high expected success rate ${ }^{[24]}$. Small $(<5 \mathrm{~mm})$ fistulas are good candidates for a primary repair, whereas larger fistulas $\left(>5 \mathrm{~mm}\right.$ ) may require a graft to cover the defect ${ }^{[11]}$. Patients who do not elect to have vaginectomy have an especially high (up to 60\%) risk for urethrocutaneous fistulas ${ }^{[11,25]}$.

Asymptomatic pseudodiverticula are very common at the point between the native urethra and the pars fixa. They can be more prominent in those with high pressure voiding from a more distal anastomotic stricture, but almost always decompress when the distal obstruction is treated. Uncommonly, these pseudodiverticula may suffer urinary tract infections from trapped urine, or cause significant post-void dribbling that may require surgical management ${ }^{[6]}$.

Meatal stenosis is a short narrowing of the most distal portion of the penile urethra and is thought to be caused by local ischemia of a watershed region, leading to contracture of the skin at the meatus ${ }^{[8]}$. This complication is generally straightforward to treat through meatoplasty with good outcomes ${ }^{[2]}$. Meatal stenosis generally does not have significant long-term urinary consequences when treated ${ }^{[24]}$.

Multiple urethral complications may occur concurrently, and many patients with urethral complications may present with numerous simultaneous urologic findings ${ }^{[7]}$. For example, in RFF phalloplasty, fistulas are often located immediately proximal to a concurrent stricture or urethral trichobezoar/hairball ${ }^{[7,14]}$.

There are several methods of creating the neourethra, but we currently use the TWT almost exclusively. Prelamination of the RFF or ALT with buccal mucosal graft or even vaginal mucosa harvested from vaginectomy has been proposed, but requires an additional staged surgery without significant improvement in outcomes ${ }^{[8,10]}$. Additionally, these grafts heal by scarring into the tissue over which they are placed, and do not seem to be an improvement over the healthy, unscarred, well-vascularized arm/leg tissue they are meant to replace. Creating an ALT neourethra out of a second free flap harvested from the forearm has also been proposed, but we and others are concerned that the addition of a second free flap (especially to the ALT surgery which only requires a pedicled flap) invites a potentially dangerous second microvascular anastomosis and may not provide any advantage to the patient $t^{[6,10]}$.

\section{Improvements in pars fixa creation}

A critical improvement in phalloplasty came from using bulbospongiosus muscle flaps as a second layer of the proximal pars fixa. It greatly decreased the rate of fistulas and strictures in this area ${ }^{[26]}$. This is an essential step in our urethral lengthening technique when vaginectomy is simultaneously performed. Additionally, we cover the more distal pars fixa with a second layer of labial minora tissue ${ }^{[26]}$. These additional layers are thought to reinforce the vascular supply of the pars fixa, and have further reduced the rate of fistulas and strictures in this area dramatically.

\section{Improvements in distal urethral anastomosis}

The anastomosis of the pars fixa with the pars pendulans is the most common site for urethral strictures and fistulas after phalloplasty ${ }^{[7,8]}$. The higher prevalence of anastomotic strictures and fistulas here is most often attributed to local ischemia in this "double watershed" zone where the edge of the neourethra portion of the flap has the poorest blood flow, and the edge of the pars fixa urethra flaps have their poorest blood supply ${ }^{[6,8]}$. 
It has been challenging to surgically create the anastomosis of the pars fixa and the pars pendulans of the neourethra in a way that maintains sufficient vascular supply, promotes healing, and minimizes these complications. It is recommended that all patients undergoing phalloplasty have the placement of a suprapubic catheter for urinary flow diversion during the initial healing stages to prevent complications ${ }^{[8]}$. This usually stays in place for 2-3 weeks postoperatively ${ }^{[11]}$. Techniques used to maximize vascularity in this troublesome area begin with donor flap choice. The RFF flaps are generally the most well-vascularized option $^{[3,6]}$. Additionally, careful dissection of the flap to preserve its blood supply is also imperative; dissection of an additional $2 \mathrm{~cm}$ rim of nearby fatty tissue that might usually be discarded and sparing the nearby dermis can help further preserve blood supply.

When attaching the pars fixa to the pars pendulans, it is important to create a tensionless closure. Methods to reduce tension in this area include creating a urethral extension from the donor flap. The anastomotic suture line can be further protected by the addition of a second well-vascularized layer over the anastomotic section of urethra using a variety of tissues. We have studied the use of the dorsal clitoris dartos tissue, which is dissected free and moved ventrally to cover the urethral suture line, with indeterminate results so far. Overlay of the urethra with the gracilis flap seems to decrease the rate of stricture and fistula formation at the cost of significant additional surgery, but has not yet been widely adopted ${ }^{[27,28]}$. Some manuscripts mention the use of pedicled rectus flaps as an additional well-vascularized layer, but we were unable to find any scientific reports describing its use ${ }^{[7]}$. While coverage with the labia majora (Martius) flap has also been suggested, we hesitate to disrupt the delicate labial majora flaps that are already used to create a neoscrotum in most patients ${ }^{[6-8]}$.

Our group has also studied using tissue engineered technology, particularly a bioactive tissue matrix allograft composed of dehydrated human amnion/chorion membrane (dHACM) (Amniofix ${ }^{\mathrm{TM}}$; MiMedx, Marietta, GA) to promote healing of the anastomotic urethra. AHACM is a scaffold that contains hundreds of functioning growth factors and cytokines, including platelet derived growth factor AA (PDGF-AA), transforming growth factor b1 (TGFb1), vascular endothelial growth factor (VEGF), fibroblast growth factor 2 (FGF-2), interleukin (IL)-4, 6, 8, and 10, and tissue inhibitor of metalloproteinase 1 and 2 in high concentrations and physiologic ratios ${ }^{[29]}$. This material has been shown to promote fibroblast and endothelial cell proliferation and support angiogenesis of the surrounding tissue ${ }^{[29]}$. In order to decrease scarring and improve healing in this vulnerable area, we have covered the neourethral anastomosis with a $12 \mathrm{~cm}$ by $2 \mathrm{~cm}$ piece of Amniofix ${ }^{\mathrm{TM}}$, with uncertain benefit to date.

\section{HEMOSTATIC AGENTS IN SCROTOPLASTY}

Dissection of the labia majora to create the neoscrotum must maximally preserve the blood supply to these flaps. These flaps have a large surface area, loose underlying connective tissue, and are closed into a spherical geometric shape that tamponades bleeding poorly. Ongoing bleeding after scrotoplasty can be clinically troublesome and result in flap necrosis, scrotal dehiscence, or urethral fistula ${ }^{[\rho]}$. Rarely, some patients may require anticoagulation after microsurgery (e.g., vessel thrombosis), which increases the risk of hematoma formation. Because of this, we routinely use a thrombin-gelatin hemostatic matrix FloSeal ${ }^{\mathrm{TM}}$ (Baxter Healthcare; Deerfield, IL) to eliminate the need for scrotal drains and have limited scrotal hematoma, with good effect.

\section{NERVE REGENERATION}

Attainment of both tactile and erogenous sensation in the neophallus is one of the principal goals of phalloplasty. With widespread adoption of microvascular techniques, a sensate neophallus is now achievable via microanastomosis of one or more flap nerves to the dorsal clitoral nerve of the clitoris ${ }^{[8]}$. The donor nerve from the flap (i.e., medial/lateral antebrachial cutaneous nerve from a RFF flap, or femoral cutaneous nerve from an ALT flap) is microanastamosed to one of the two dominant dorsal clitoral nerve branches. 
Additional nerves that are present in the flap may also be anastomosed to the ilioinguinal nerve, further improving light touch sensation to the neophallus ${ }^{[11,12]}$.

The technique of microsuturing the epineurium has imperfect functional outcomes ${ }^{[30]}$. The axons from the distal natal nerve must traverse the anastomosis and reach the proximal segment of the donor nerve. Any anastomotic site scarring or fibrosis may create a mechanical impediment to the axons and limit reinnervation outcomes ${ }^{[30]}$. In addition to joining the aponeuroses with several sutures, we augment the nerve repair with a fibrin sealant (Tisseel ${ }^{\circ}$; Baxter Healthcare; Deerfield, IL or an equivalent product). This serves to mechanically protect the nerve repair during the remaining surgery, and from pulling during patient movement ${ }^{[31,32]}$. Our patients have had favorable nerve regeneration outcomes after phalloplasty; about $80 \%$ achieve erogenous sensation in the neophallus, and up to $95 \%$ achieve light touch sensation in the neophallus. A notable $5 \%$ have no sensation to the neophallus whatsoever.

In order to further increase the speed and outcome of nerve regeneration, we have studied the use of nerve conduit wraps intended for peripheral nerve repair, such as the Axoguard ${ }^{\mathrm{TM}}$ Nerve Protector Wrap (Axogen; Alachua, FL) to augment the standard end-to-end surgical technique. The ideal nerve wrap is nonimmunogenic, strong enough to resist degradation and compression, and prevents both scarring and nerve adhesion formation ${ }^{[33]}$. The Axoguard ${ }^{\mathrm{TM}}$ implant is a sheath made of porcine extracellular matrix (ECM) from small intestinal submucosa ${ }^{[33,34]}$. Its acellular matrix is composed of collagen, fibronectin, growth factors, glycosaminoglycans, proteoglycans, and glycoproteins, all which may promote neural tissue revascularization through the induction of cellular proliferation/differentiation as well as the deposition of host ECM components ${ }^{[33,34]}$. It provides a physical barrier around the anastomosis, potentially protecting it from cellular infiltration and allowing the nerve to glide normally during movement ${ }^{[33]}$. With other surgical indications, it has been shown to decrease nerve scarring during healing, with favorable functional outcomes ${ }^{[33,34]}$. We will continue to investigate the role of these ECM nerve connection sheaths to see if they will improve the efficiency of nerve regeneration over fibrin sealant alone.

\section{POSTOPERATIVE FLAP MONITORING \& FLAP COMPLICATIONS}

The immediate postoperative healing period is crucial to phalloplasty flap survival. Notable potential complications include infection, hematoma, wound dehiscence, urethral loss, partial phallic loss, and full phallic loss ${ }^{[9]}$. These complications are most frequently due to vascular compromise, often venous congestion leading to potentially irreversible tissue injury ${ }^{[35-37]}$. The flaps used in phalloplasty pose an additional challenge: they are much larger than most free flaps used for other tissue coverage indications, are freely mobile, are anchored only at the base, are in a dependent position, and are positioned close to a natural flexion point of the body ${ }^{[6,9]}$. Partial and total phallic loss are devastating complications for both the patient and surgeon.

An estimated $1 \%-9 \%$ of free flaps across all indications are lost due to vascular compromise ${ }^{[4,35-38]}$ with an estimated $95 \%$ of losses occurring during the first $24-72 \mathrm{~h}$ after surgery ${ }^{[37,39]}$. At our center, the rate of flap compromise requiring reoperation after RFF phalloplasty is less than $1 \%$, and much lower after ALT phalloplasty. We currently have a $0 \%$ rate of acute, total neophallus loss. Even in those rare patients with arterial/venous thrombosis in the early postoperative period, in our cohort of $>750$ phalloplasty patients we have not lost any flaps to acute graft thrombosis. Similarly, clinically significant partial flap loss is rare at our center, although instances of partial flap compromise may contribute to urethral stricture and/or fistula development ( $20 \%$ and $22 \%$ at our center, respectively). This is likely because of several factors: our use of continuous flap oxygen monitoring, attentive expert bedside nursing, an on-call team of two surgeons available at all times for emergency revision surgery, and the optimization of techniques made possible by a large volume experience. 


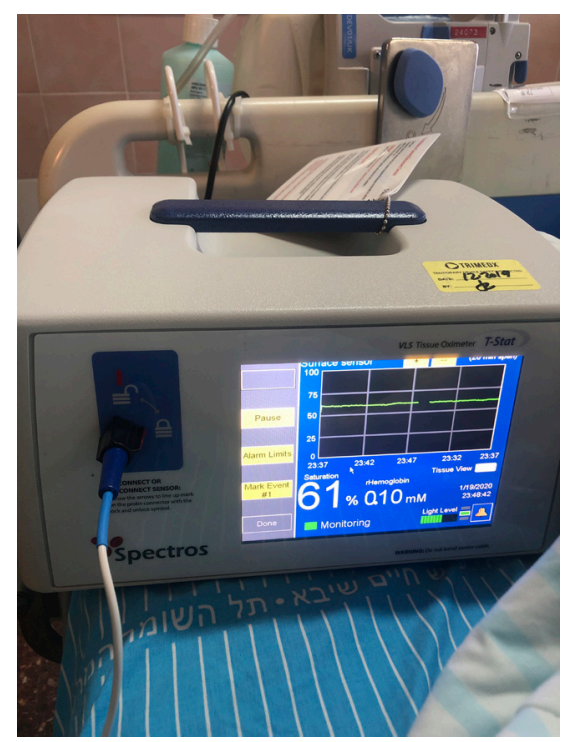

Figure 8. We use the T-Stat ${ }^{\text {TM }}$ VLS Tissue Oximeter (Spectros; Portola Valley, CA) for postoperative flap monitoring in all patients, and sometimes for intraoperative decision-making. It provides continuous quantitative measurements of total hemoglobin concentration [Hgb] and hemoglobin oxygen saturation

Early identification of vascular compromise is directly correlated with successful flap salvage rates ${ }^{[35-37,39]}$. Typical postoperative flap monitoring includes bedside physical exam in combination with handheld Doppler monitoring of the arterial signal ${ }^{[3,37,39]}$. The clinical exam includes flap skin color, capillary refill, and surface temperature ${ }^{[1,37]}$. If necessary, pinprick bleeding may be used, although we do not routinely use this at our center. Cutaneous handheld Dopplers are used to detect intravascular arterial and venous blood flow $^{[37]}$. These checks occur every 1-2 $\mathrm{h}$ at the bedside for the first few days postoperatively. Flaps that are concerning for incipient vascular compromise are monitored even more frequently. While clinical evaluation and Doppler alone are generally effective and have negligible additional $\operatorname{cost}^{[37]}$, there are drawbacks to this method. Clinical examination can vary between observers, require trained staff, and may not be able to detect vascular compromise until relatively late physical findings become apparent ${ }^{[36]}$. At that time, damage to the flap microvasculature may be irreversible. Additionally, while Doppler is sensitive to arterial compromise, it is less sensitive to venous compromise ${ }^{[35,36]}$. This is problematic given that venous congestion is the most common cause of flap failure ${ }^{[35-37]}$. Furthermore, the strength of the arterial Doppler signal does not correlate with sufficient oxygen delivery to the flap and loss of the Doppler signal can be a relatively late finding ${ }^{[35,38]}$. Neither clinical examination nor handheld Dopplers are able to continuously monitor the integrity of the flap, which could delay detection of vascular compromise ${ }^{[35,36]}$.

The ideal flap monitoring system is noninvasive, continuous, reliable, sensitive, and easy to interpret ${ }^{[37,39]}$. Our group uses the T-Stat ${ }^{\mathrm{TM}}$ VLS Tissue Oximeter (Spectros; Portola Valley, CA) to augment routine bedside

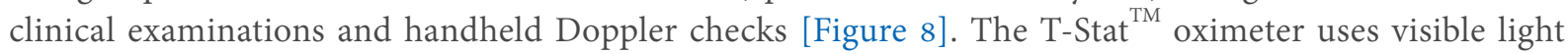
spectroscopy (VLS) to continuously monitor total hemoglobin concentration [Hgb] as well as hemoglobin saturation $\left(\mathrm{StO}_{2}\right)$ of tissue at the capillary level in real time ${ }^{[35,36]}$. [ Hgb] approximates the total blood volume in the flap, and an increased value indicates obstruction of venous drainage ${ }^{[36]}$. StO $\mathrm{S}_{2}$ measurements correlate directly with arterial tissue perfusion, and a decreased value indicates diminished arterial inflow from an arterial thrombus or is a later finding in the case of venous drainage obstruction ${ }^{[36]}$. In this way, VLS monitoring is able to continuously quantify the arterial inflow, venous outflow, and perfusion status of the flap $^{[36]}$.

Multiple groups have demonstrated an initial gradual increase in $\mathrm{StO}_{2}$ detected with VLS in the first $\sim 8 \mathrm{~h}$ after surgery before the value stabilizes, attributable to mild ischemia-reperfusion injury ${ }^{[35,36]}$. The mean 


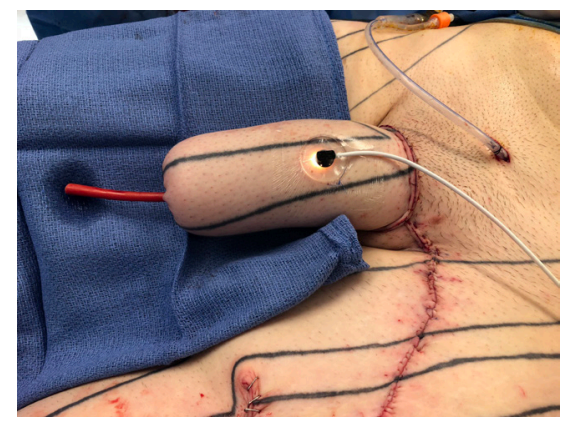

Figure 9. Placement of the T-Stat ${ }^{\mathrm{TM}}$ VLS Tissue Oximeter (Spectros; Portola Valley, CA) probe for monitoring of the vascular integrity of the phalloplasty flap in the immediate postoperative period

$\mathrm{StO}_{2}$ has been reported as $49 \%-57 \%$ with a range of $40 \%-75 \%$ regardless of flap type ${ }^{[35,36,39]}$. There are no established criteria for oximeter detection of flap compromise, although one group proposed using either a 20-point drop in $\mathrm{StO}_{2}$ within a 1 -h period or an absolute reading less than $30 \%$ to indicate vascular compromise ${ }^{[38]}$. Following the trends in $[\mathrm{Hgb}]$ and $\mathrm{StO}_{2}$ over time is more valuable than the absolute readings due to the heterogeneity of vessels in the flap ${ }^{[38]}$. Some advantages of the $\mathrm{T}^{2} \mathrm{Stat}^{\mathrm{TM}}$ oximeter is the ability to set an alarm when user-specified criteria are met, and the ability to mirror quantitative outputs to the surgeon's smartphone app in real time for remote monitoring ${ }^{[36,38]}$. Previous groups have found that VLS continuous monitoring resulted in earlier detection of flap vascular compromise 1-3 $\mathrm{h}$ or more before clinical examination or Doppler findings were apparent ${ }^{[36,39]}$.

We use the T-Stat ${ }^{\mathrm{TM}}$ oximeter in all of our patients and consider it to be a nonnegotiable requirement at our center for safe flap surgery. The sensor probe is placed on the neophallus by the surgeon at the conclusion of the operation and remains in place until the patient is discharged [Figure 9].

Ultimately, flap monitoring and protective measures continue outside of the immediate postoperative period. After discharge, patients are instructed to minimize bending at the waist of more than $20^{\circ}$ for 4 weeks to avoid compression of the vascular supply to the neophallus, limit walking for 4 weeks, avoid prolonged sitting, maintain excellent genital hygiene, and to keep the neophallus positioned at $90^{\circ}$ to the body to minimize flap-related complications ${ }^{[9]}$.

\section{CLINICAL PATHWAYS}

Clinical pathways (CPWs) are tools to translate evidence-based medicine to a clinical setting for a specific clinical situation. The main goal of a CPW is to provide high quality care through aligning clinical practice with guideline recommendations. At the same time, a CPW also seeks to minimize economic measures, such as healthcare costs, resource allocation, and length of stay ${ }^{[40]}$. CPWs have been used in clinical practice internationally since the 1980s, and an estimated $80 \%$ of hospitals in the US have implemented CPWs as of $2003^{[40,41]}$. A 2012 Cochrane Review showed that CPW implementation consistently reduced in-hospital complications and improved documentation without increasing patients' length of stay or healthcare costs ${ }^{[40]}$. The majority of studies reported that CPWs decreased in-hospital complications for surgical procedures ${ }^{[40]}$.

We use a robust, standardized, 5-day inpatient CPW for all phalloplasty patients. The pathway covers dayby-day flap monitoring requirements, clinical staffing level (ICU, med-surg step-down, or general medsurg nursing care), scheduled and as-needed (PRN) medications, activity level, diet, and more. In this way, order sets are standardized between patients, decreasing the potential for errors and avoiding ad hoc orders placed for each individual. Nursing expectations for daily progress is also standardized. Patients who are not meeting the expected milestones are identified early and receive extra diagnostic/therapeutic attention. 
Finally, our patients' understanding of the day-by-day hospital course is greatly improved. Patients are given individualized treatments, especially when encountering complications, but our CPW allows patient, physician and nursing to be aligned as to each day's expectations and treatments. Because of the predictable safety profile provided by our post phalloplasty CPW, most phalloplasty patients can be safely discharged 5 days after the operation at our center.

\section{CONCLUSION}

We have described some of the more common postoperative complications and have summarized some of the established surgical techniques in the literature to date to address these complications. We have also described several more experimental techniques, many of which include promising new technology to further optimize the results of phalloplasty. Each of these techniques represents a modest but important improvement towards the goal of a safe and reproducible surgery with optimal results and minimized risk of complications.

We and others endeavor to constantly evaluate and improve the steps of this surgery and investigate new concepts and materials in this field in an ongoing effort to further improve outcomes. We have had good success with these techniques at our high-volume center, although more thorough investigation and quantification of these patient outcomes is needed. Ultimately, with continued innovation and sharing of improved surgical techniques, it may be possible to better standardize care and improve the aesthetic and functional outcomes of this incredibly complex and increasingly common surgery.

\section{DECLARATIONS}

\section{Authors' contributions}

Made substantial contribution to the conception and design of the study and performed data analysis and interpretation: Carter EE, Crane CN, Santucci RA

\section{Availability of data and materials}

Not applicable.

\section{Financial support and sponsorship}

None.

\section{Conflicts of interest}

All authors declared that there are no conflicts of interest.

\section{Ethical approval and consent to participate}

An informed consent to participate was obtained from the patients.

\section{Consent for publication}

A written informed consent for publication was obtained.

\section{Copyright}

(c) The Author(s) 2020.

\section{REFERENCES}

1. Frey JD, Poudrier G, Chiodo MV, Hazen A. An update on genital reconstruction options for the female-to-male transgender patient: a review of the literature. Plast Reconstr Surg 2017;139:728-37.

2. Özer M, Pigot GLS, Bouman MB, van de Grift TC, Elfering L, et al. Development of a decision aid for genital gender-affirming surgery in transmen. J Sex Med 2018;15:1041-8. 
3. Hadj-Moussa M, Agarwal S, Ohl DA, Kuzon WM Jr. Masculinizing genital gender confirmation surgery. Sex Med Rev 2019;7:141-55.

4. Remington AC, Morrison SD, Massie JP, Crowe CS, Shakir A, et al. Outcomes after phalloplasty: do transgender patients and multiple urethral procedures carry a higher rate of complication? Plast Reconstr Surg 2018;141:220e-9.

5. Ascha M, Massie JP, Morrison SD, Crane CN, Chen ML. Outcomes of single stage phalloplasty by pedicled anterolateral thigh flap versus radial forearm free flap in gender confirming surgery. J Urol 2018;199:206-14.

6. Heston AL, Esmonde NO, Dugi DD 3rd, Berli JU. Phalloplasty: techniques and outcomes. Transl Androl Urol 2019;8:254-65.

7. Dy GW, Granieri MA, Fu BC, Vanni AJ, Voelzke B, et al. Presenting complications to a reconstructive urologist after masculinizing genital reconstructive surgery. Urology 2019;132:202-6.

8. Dy GW, Sun J, Granieri MA, Zhao LC. Reconstructive management pearls for the transgender patient. Curr Urol Rep 2018;19:36.

9. Esmonde N, Bluebond-Langner R, Berli JU. Phalloplasty flap-related complication. Clin Plast Surg 2018;45:415-24.

10. Danker S, Esmonde N, Berli JU. "Staging” in phalloplasty. Urol Clin North Am 2019;46:581-90.

11. Chen ML, Reyblat P, Poh MM, Chi AC. Overview of surgical techniques in gender-affirming genital surgery. Transl Androl Urol 2019;8:191-208.

12. Morrison SD, Shakir A, Vyas KS, Kirby J, Crane CN, et al. Phalloplasty: a review of techniques and outcomes. Plast Reconstr Surg 2016;138:594-615.

13. Gravvanis AI, Tsoutsos DA, Iconomou T, Gremoutis G. The use of integra artificial dermis to minimize donor-site morbidity after suprafascial dissection of the radial forearm flap. Microsurgery 2007;27:583-7.

14. Pigot GLS, Belboukhaddaoui S, Bouman MB, Meuleman EJH, de Boer EM, et al. Effectiveness of preoperative depilation of the urethral donor site for phalloplasty: neourethral hair growth and its effects on voiding. Eur Urol Focus 2020;6:770-5.

15. Bryson C, Honig SC. Genitourinary complications of gender-affirming surgery. Curr Urol Rep 2019;20:31.

16. Gao Y, Maurer T, Mirmirani P. Understanding and addressing hair disorders in transgender individuals. Am J Clin Dermatol 2018;19:517-27.

17. Carney MJ, Samra F, Momeni A, Bauder AR, Weissler JM, et al. Anastomotic technique and preoperative imaging in microsurgical lower-extremity reconstruction: a single-surgeon experience. Ann Plast Surg 2020;84:425-30.

18. Wirthmann A, Finke JC, Giovanoli P, Lindenblatt N. Long-term follow-up of donor site morbidity after defect coverage with Integra following radial forearm flap elevation. Eur J Plast Surg 2014;37:159-66.

19. Chang DK, Louis MR, Gimenez A, Reece EM. The basics of integra dermal regeneration template and its expanding clinical applications. Semin Plast Surg 2019;33:185-9.

20. Rudnicki PA, Purt B, True D, Siordia H, Lohmeier S, et al. Single-stage composite skin reconstruction using a dermal regeneration template. Plast Reconstr Surg Glob Open 2020;8:e2622.

21. Demiri E, Papaconstantinou A, Dionyssiou D, Dionyssopoulos A, Kaidoglou K, et al. Reconstruction of skin avulsion injuries of the upper extremity with integra $(\mathbb{R})$ dermal regeneration template and skin grafts in a single-stage procedure. Arch Orthop Trauma Surg 2013;133:1521-6.

22. Koenen W, Felcht M, Vockenroth K, Sassmann G, Goerdt S, et al. One-stage reconstruction of deep facial defects with a single layer dermal regeneration template. J Eur Acad Dermatol Venereol 2011;25:788-93.

23. Papa G, Pangos M, Renzi N, Ramella V, Panizzo N, et al. Five years of experience using a dermal substitute: indications, histologic studies, and first results using a new single-layer tool. Dermatol Surg 2011;37:1631-7.

24. Santucci RA. Urethral complications after transgender phalloplasty: strategies to treat them and minimize their occurrence. Clin Anat 2018;31:187-90.

25. Al-Tamimi M, Pigot GL, van der Sluis WB, van de Grift TC, Mullender MG, et al. Colpectomy significantly reduces the risk of urethral fistula formation after urethral lengthening in transgender men undergoing genital gender affirming surgery. J Urol 2018;200:1315-22.

26. Massie JP, Morrison SD, Wilson SC, Crane CN, Chen ML. Phalloplasty with urethral lengthening: addition of a vascularized bulbospongiosus flap from vaginectomy reduces postoperative urethral complications. Plast Reconstr Surg 2017;140:551e-8.

27. Gilbert DA, Winslow BH, Gilbert DM, Jordan GH, Horton CE. Transsexual surgery in the genetic female. Clin Plast Surg 1988;15:47187.

28. Salgado CJ, Nugent AG, Moody AM, Chim H, Paz AM, et al. Immediate pedicled gracilis flap in radial forearm flap phalloplasty for transgender male patients to reduce urinary fistula. J Plast Reconstr Aesthet Surg 2016;69:1551-7.

29. Maan ZN, Rennert RC, Koob TJ, Januszyk M, Li WW, et al. Cell recruitment by amnion chorion grafts promotes neovascularization. J Surg Res 2015;193:953-62.

30. Sarhane KA, Ibrahim Z, Martin R, Krick K, Cashman CR, et al. Macroporous nanofiber wraps promote axonal regeneration and functional recovery in nerve repair by limiting fibrosis. Acta Biomater 2019;88:332-45.

31. Narakas A. The use of fibrin glue in repair of peripheral nerves. Orthop Clin North Am 1988;19:187-99.

32. Childe JR, Regal S, Schimoler P, Kharlamov A, Miller MC, et al. Fibrin glue increases the tensile strength of conduit-assisted primary digital nerve repair. Hand (N Y) 2018;13:45-9.

33. Siemionow M, Uygur S, Ozturk C, Siemionow K. Techniques and materials for enhancement of peripheral nerve regeneration: a literature review. Microsurgery 2013;33:318-28.

34. Kehoe S, Zhang XF, Boyd D. FDA approved guidance conduits and wraps for peripheral nerve injury: a review of materials and efficacy. Injury 2012;43:553-72.

35. Fox PM, Zeidler K, Carey J, Lee GK. White light spectroscopy for free flap monitoring. Microsurgery 2013;33:198-202.

36. Mericli AF, Wren J, Garvey PB, Liu J, Butler CE, et al. A prospective clinical trial comparing visible light spectroscopy to handheld 
doppler for postoperative free tissue transfer monitoring. Plast Reconstr Surg 2017;140:604-13.

37. Kohlert S, Quimby AE, Saman M, Ducic Y. Postoperative free-flap monitoring techniques. Semin Plast Surg 2019;33:13-6.

38. Chao AH, Meyerson J, Povoski SP, Kocak E. A review of devices used in the monitoring of microvascular free tissue transfers. Expert Rev Med Devices 2013;10:649-60.

39. Cornejo A, Rodriguez T, Steigelman M, Stephenson S, Sahar D, et al. The use of visible light spectroscopy to measure tissue oxygenation in free flap reconstruction. J Reconstr Microsurg 2011;27:397-402.

40. Rotter T, Kinsman L, James E, Machotta A, Willis J, et al. The effects of clinical pathways on professional practice, patient outcomes, length of stay, and hospital costs: Cochrane systematic review and meta-analysis. Eval Health Prof 2012;35:3-27.

41. Müller MK, Dedes KJ, Dindo D, Steiner S, Hahnloser D, et al. Impact of clinical pathways in surgery. Langenbecks Arch Surg 2009;394:31-9. 